\title{
Coherent Acoustic Phonon Oscillations in Semiconductor Multiple Quantum Wells with Piezoelectric Fields
}

\author{
Chi-Kuang Sun,* Jian-Chin Liang, and Xiang-Yang Yu \\ Department of Electrical Engineering and Graduate Institute of Electro-Optical Engineering, \\ Taipei 10617, Taiwan, Republic of China
}

(Received 16 August 1999)

\begin{abstract}
Large coherent acoustic phonon oscillations were demonstrated using InGaN/GaN multiple quantum wells with piezoelectric fields. With UV femtosecond pulse excitation, photogenerated carriers screened the piezoelectric field and initiated the displacive coherent phonon oscillations. The specific phonon frequency was selected by the coupling between the periodic carrier distribution and the corresponding acoustic phonon mode. The induced acoustic phonon oscillation resulted in piezoelectric field modulation and then caused absorption variation through the quantum confined Franz-Keldysh effect. The wave vector uncertainty due to the finite sample width was found to determine the observed dephasing time.
\end{abstract}

PACS numbers: 78.47.+p, 42.65.Re, 43.35.+d, 77.65.-j

Progress in femtosecond lasers and ultrafast spectroscopy technology has enabled us to generate and directly observe the coherent oscillation of phonon modes [1-9]. Oscillation changes in optical properties have been observed in GaAs [1,2], Ge [3], and a number of other materials $[2,4,5]$. In semiconductors, carriers can be excited by ultrashort pump pulses in a spatial area much larger than one lattice unit cell, and the excited carrier populations (with wave vector $q \cong 0$ ) are coupled to the corresponding optical phonon modes (also with $q \cong 0$ ) [6]. This optically excited coherent optical phonon mode can behave like classical oscillators and have nonzero time-dependent displacement. This is different from spontaneous emission of phonons which is an incoherent process that populates the phonon modes with the expectation value of each mode being zero. The corresponding modulation of the material dielectric constant, due to coherent oscillation of optical phonons, could then be observed by changes in the intensities of transmitted or reflected probe light pulses.

For acoustic phonons, semiconductor superlattices exhibit zone folding of the acoustic branches within the mini-Brillouin zone due to the artificial periodicity, enabling the observation of coherent oscillation of the zonefolded acoustic phonons in AlAs/GaAs superlattices [8,9]. However, the observed reflection modulation $\Delta R / R$ was extremely small, on the order of $10^{-5}-10^{-6}$ [9]. The detection of the coherent acoustic modes was based on the modulation of the interband transitions via the acoustic deformation potential [9].

In this paper, we report our studies of the coherent acoustic phonon oscillation in semiconductor multiple quantum wells (MQWs) with strain-induced piezoelectric field. Strong coherent acoustic phonon oscillation can be easily generated with induced transmission/absorption modulation on the order of $10^{-2}-10^{-3}$. The oscillation frequency, on the order of $\mathrm{THz}$, can be varied by tuning the sample period width. This strong oscillation can be induced and probed by almost any above band gap photons, indicating a different physical mechanism from those of previous reports [8,9]. With femtosecond pulse excitation, the photoexcited carriers screened the piezoelectric field and thus initiated the coherent acoustic phonon oscillation via the coupling between the periodic carrier distribution and the corresponding acoustic phonon mode. With a photoexcited carrier density on the order of $1 \times 10^{19} \mathrm{~cm}^{-3}$, the induced strain modulation can be on the order of a fraction of a percent. The resulted modulation of piezoelectric field would then cause absorption modulation through the quantum-confined Franz-Keldysh effect. A fast dephasing time was observed for the coherent acoustic phonon oscillations. In contrast to previous studies, our observed fast dephasing time was attributed to the finite sample width that caused a broadening in the linewidth of the corresponding phonon wave vectors. This easily accessible large oscillation opens the way to the studies of the properties of acoustic phonons and their interactions with surrounding carriers in semiconductors. With such a large transmission modulation due to coherent acoustic phonon oscillation, this interesting phenomena not only cannot be neglected, but can be applied to practical high-speed optoelectronic applications. Because of the acoustic phonon dispersion relation, tunable oscillation frequencies ranging from low frequency up to several $\mathrm{THz}$ can then be easily generated with one material system.

The experiments were performed at room temperature on 14 periods $\mathrm{InGaN} / \mathrm{GaN}$ MQWs [10]. The recent demonstration of high-brightness light-emitting diodes and laser diodes with InGaN MQW active regions [11] has established the III-V nitrides as key materials for optoelectronics operating in the green-UV wavelength range. Because of the large piezoelectric constants along the [0001] orientation group III nitrides, a strain-induced piezoelectric field on the order of $\mathrm{MV} / \mathrm{cm}$ is expected. This large piezoelectric field would play an important role in the optical properties of (0001)-oriented InGaN MQWs due to the quantum-confined Stark effect (QCSE) [10,12]. The barrier widths of all samples were $43 \AA$ while the well widths were varied from 12 to $62 \AA$. The In composition 
in the MQWs was determined to be between $6 \%-10 \%$ by $\mathrm{x}$-ray diffraction. Room temperature photoluminescence (PL) study and absorption measurements indicated that the band gaps of MQWs were between 390-430 nm $(3.177-2.88 \mathrm{eV})$ for $12-62 \AA$ well-width MQWs. The crystal structures were wurzite.

Femtosecond transmission pump-probe measurements were performed using a Kerr-Lens Mode-locked Ti:sapphire laser. The laser output pulses were frequency doubled using a BBO crystal to reach the band gap energy of the InGaN/GaN MQWs. The frequency-doubled pulses had a pulse width of $180 \mathrm{fs}$ at a wavelength of $390 \mathrm{~nm}$ measured by a two-photon-absorption-type pump-probe autocorrelation in a bulk GaN thin film [13]. The femtosecond pump pulses photoexcited carriers in the MQWs. Because of the periodic distribution of the photoexcited carrier population, carriers with wave vector $q=2 \pi / a$, where $a$ is the MQW period width, were thus created. Coherent oscillation, with the selected acoustic phonon mode corresponding to the carrier wave vector $q$, can then be initiated according to the theory of Kuznetsov and Stanton [6]. Similar principles have previously been applied to the generation of $\mathrm{MHz}-\mathrm{GHz}$ coherent acoustic waves through periodic optical fields [14] or multilayer structures [15]. Because of the piezoelectric-induced QCSE, the generated electron and hole wave functions were separated in space and thus created a photocarrier screening field that would screen out part of the piezoelectric field. With a new equilibrium position due to the photocarrier screening of the piezoelectric field, displacive oscillations of the coherent acoustic phonons with nonzero time-dependent displacement should then be induced. Figure 1 shows the measured probe transmission changes as a function of probe delay for the $50 \AA$ sample at wavelengths of $390 \mathrm{~nm}$ $(3.177 \mathrm{eV})$ and $365 \mathrm{~nm}(3.39 \mathrm{eV})$. The average incident pump powers were $20 \mathrm{~mW}$. The average $2 \mathrm{D} / 3 \mathrm{D}$ photocarrier densities were $9 \times 10^{12} \mathrm{~cm}^{-2} / 1.8 \times 10^{19} \mathrm{~cm}^{-3}$ and $1.5 \times 10^{13} \mathrm{~cm}^{-2} / 3 \times 10^{19} \mathrm{~cm}^{-3}$ for 390 and $365 \mathrm{~nm}$ traces, respectively. After the pump excited carriers and caused transient transmission increases at zero time delay, a clear cosine-like transmission oscillation could be observed on top of the background signals of carrier cooling dynamics [16]. Large transmission modulation $\Delta T / T$ on the order of $10^{-2}$ can be observed. This optical property modulation is $\sim 10^{3}$ times larger than previous coherent-phonon-induced optical property modulation in III-V semiconductor systems $[1,2,8,9]$. Within our experimental resolution, the observed oscillation frequency was found to be independent of pump/probe photoenergy or pump fluence. The phase components of the cosine arguments were always close to either zero or $\pi$, suggesting the displacive nature of the oscillations [4]. The observed cosine-like oscillation agreed with the picture that a new equilibrium position was set up for the lattice system by the photocarrier screening of the strain-induced piezoelectric field.
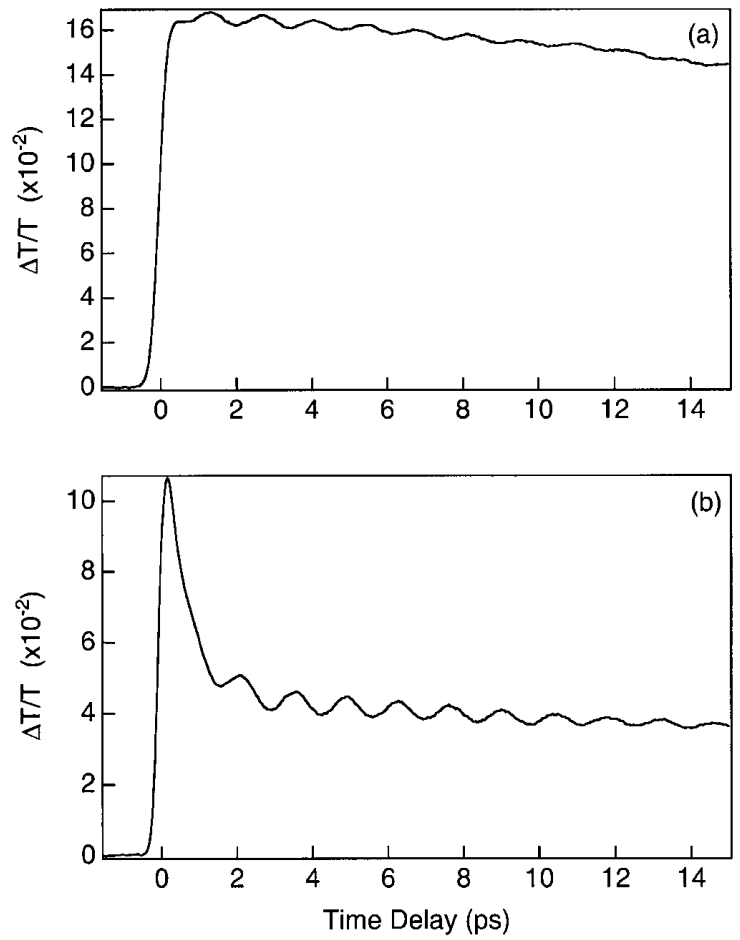

FIG. 1. Measured transient transmission changes vs probe delay for $50 \AA / 43 \AA \mathrm{InGaN} / \mathrm{GaN}$ MQW with an average pump power of $20 \mathrm{~mW}$. (a) Laser wavelength was $390 \mathrm{~nm}$. (b) Laser wavelength was $365 \mathrm{~nm}$. Cosine-like oscillation can be observed on top of the background signals. Please notice the $\pi$ phase shift between traces (a) and (b). Oscillation dephasing time was on the order of $8 \mathrm{ps}$.

Since the MQWs were grown on $c$-plane sapphires, we attribute this large oscillation to coherent longitudinalacoustic (LA) phonon oscillation in the QW growth direction ( $c$ axis). The observed oscillation period for $50 \AA \mathrm{MQW}$ was $1.38 \mathrm{ps}$, corresponding to a frequency of $0.72 \mathrm{THz}$. With different MQW period widths, we should expect different oscillating acoustic phonon modes. We have repeated our experiments on samples with different well widths. The oscillation frequency varied from $1.23 \mathrm{THz}$ for QW period of $55 \AA$ (12 ̊ well) to $0.66 \mathrm{THz}$ for QW period of $105 \AA(62 \AA$ well). Figure 2 plots the observed oscillation angular frequency $\omega$ ( $2 \pi \times$ frequency, open circles) vs photocarrier wave vector $q$, which is determined by the MQW period width, for experiments with different photoenergies and of different samples. A linear relation between angular frequency and wave vector can be observed. The slope of the observed linear relation, which is $6820 \mathrm{~m} / \mathrm{s}$ (dotted line), should correspond to the sound velocity in the [0001] direction for the LA mode in wurzite $\mathrm{In}_{0.06} \mathrm{GaN}$. This value agrees well with the LA mode $c$ axis sound velocity of $\mathrm{GaN}$ of $6620 \pm 220 \mathrm{~m} / \mathrm{s}$ which can be derived from the room temperature elastic stiffness constant $c_{33}$ [17].

After the coherent phonon oscillation was activated by photocarrier screening, the induced coherent oscillation with nonzero time-dependent displacement would 


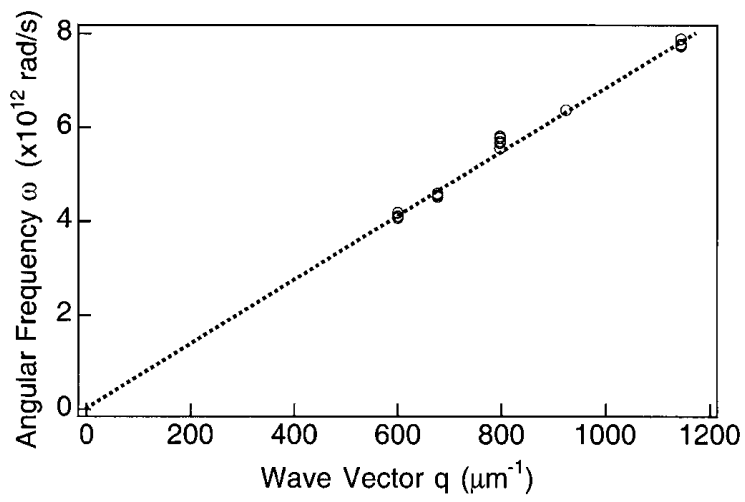

FIG. 2. Measured angular frequency of oscillation (open circles) vs carrier wave vector for different MQWs. The dashed line corresponds to a sound velocity of $6820 \mathrm{~m} / \mathrm{s}$.

then modulate the strain-induced piezoelectric field and would result in absorption changes through the quantumconfined Franz-Keldysh effect [18], which describes the electric-field dependent absorption in quantum-well structures. Figure 3 shows the calculated normalized absorption change $\Delta \alpha / \alpha$ due to $1 \%$ strain modulation in the bulk $\mathrm{In}_{0.06} \mathrm{GaN}$. For simplicity, the $\mathrm{InGaN}$ layer was assumed to be fully strained to lattice match the $\mathrm{GaN}$ substrate prior to the modulation. Both absorption changes due to strain variation and strain-induced piezoelectric field variation were considered. Recent studies indicated that the critical thickness of $\operatorname{In}_{0.1} \mathrm{GaN}$ is greater than $400 \AA$ [10], while the free exciton Bohr radius of $\mathrm{GaN}$ is on the order of $34 \AA$ [10], which is less than the well width of parts of our samples. A sign change due to Franz-Keldysh oscillation can be observed in Fig. 3 around $3.3 \mathrm{eV}(\sim 375 \mathrm{~nm})$. For smaller well-width samples with quantization effect considered, we would then expect a blueshift in the zero-crossing energy. This conforms to the observed sign changes (or $\pi$ phase shift)

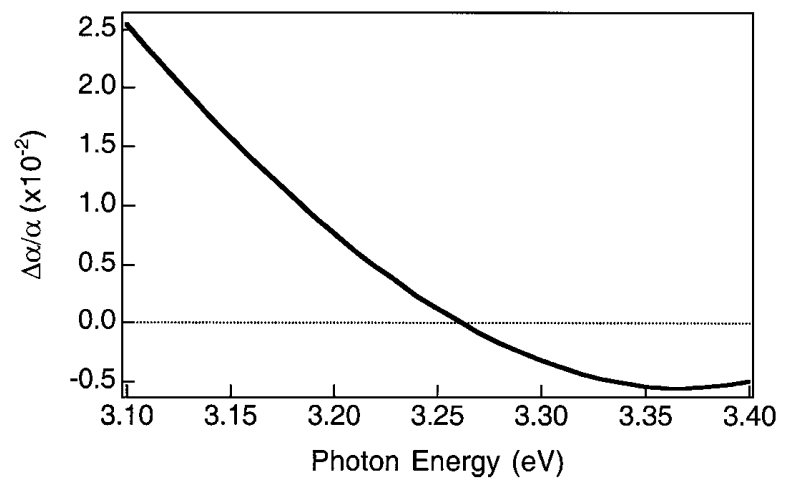

FIG. 3. Calculated absorption modulation $\Delta \alpha / \alpha$ due to $1 \%$ strain variation in the bulk $\operatorname{In}_{0.06} \mathrm{GaN}$. The InGaN layer was assumed to be fully strained to lattice match the GaN substrate prior to the modulation. Both absorption changes due to strain variation and strain-induced piezoelectric field variation were considered. $\Delta \alpha / \alpha$ changes sign around $3.3 \mathrm{eV}$ due to the FranzKeldysh effect. of the cosine oscillations in Fig. 1 for two different probe photon energies of 3.177 and $3.39 \mathrm{eV}$. Figure 4 plots the measured normalized absorption change $\Delta \alpha / \alpha$ due to coherent oscillation versus probe photon energy for 36 and $50 \AA$ samples with a fixed average photoexcitation carrier density of $1 \times 10^{19} \mathrm{~cm}^{-3}$. Most of our experiments could create an even higher carrier density. While the observed $\Delta \alpha / \alpha$ changed signs as predicted by the Franz-Keldysh effect shown in Fig. 3, a blueshift in the zero-crossing energy was also observed for the narrower well width sample, as expected by the quantization effect. By comparing Fig. 4 with Fig. 3, the strain modulation on the order of a fraction of a percent can then be estimated.

In Fig. 1, it is interesting to notice the fast dephasing time observed in our experiments. However, this fast dephasing time should not reflect the acoustic phonon lifetimes of these specific LA phonon modes in GaN. We attribute our observed fast dephasing time to finite sample thickness due to the limited number (14) of quantum wells. For the periodic carrier distribution generated in a limited space, the corresponding wave vector $q$ will have a magnitude uncertainty $\Delta q$ due to the finite sample width, which would result in a sinc-like (instead of a delta-like) distribution in the corresponding $q$ space. The initiated coherent acoustic phonon oscillation will then have an uncertainty $\Delta \omega$ in the angular frequency due to the uncertainty $\Delta q$ in the corresponding wave vector with $\Delta \omega / \omega=\Delta q / q$. This $\Delta \omega$ linewidth will then result in a fast dephasing of the oscillation. Assuming a single exponential decay of the cosine oscillation with a decay time constant of $\tau$ and an oscillation period of $T$, a simple relation of $\tau=6.38 T$ can then be derived with the sample thickness equal to 14 times of the QW period width. Figure 5 plots the dephasing time vs oscillation period for 5 different samples and for different photon energies. The dephasing times were obtained by using a simple convolution fit assuming a single exponential decay of the cosine oscillation of the form $\cos (2 \pi t / T) e^{-t / \tau} u(t)$, where $u(t)$ is the step function.

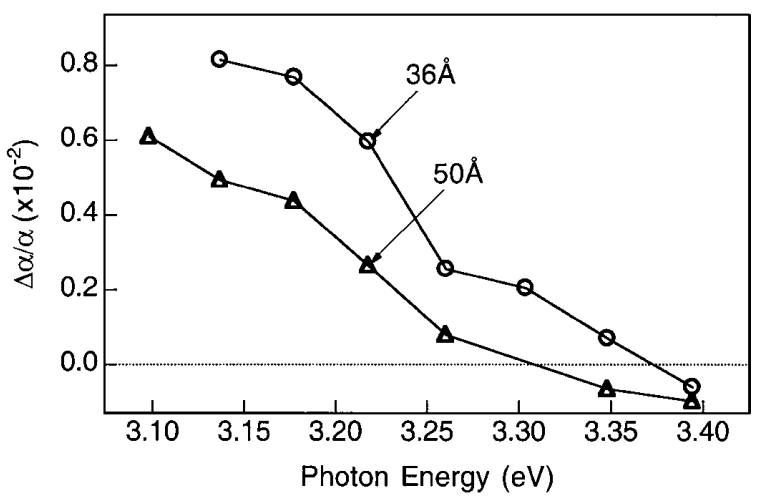

FIG. 4. Coherent oscillation induced absorption modulation $\Delta \alpha / \alpha$ vs pump/probe photon energy for $36 \AA$ (open circles) and $50 \AA$ (open triangles) samples. The photoexcited carrier densities were $1 \times 10^{19} \mathrm{~cm}^{-3}$. 


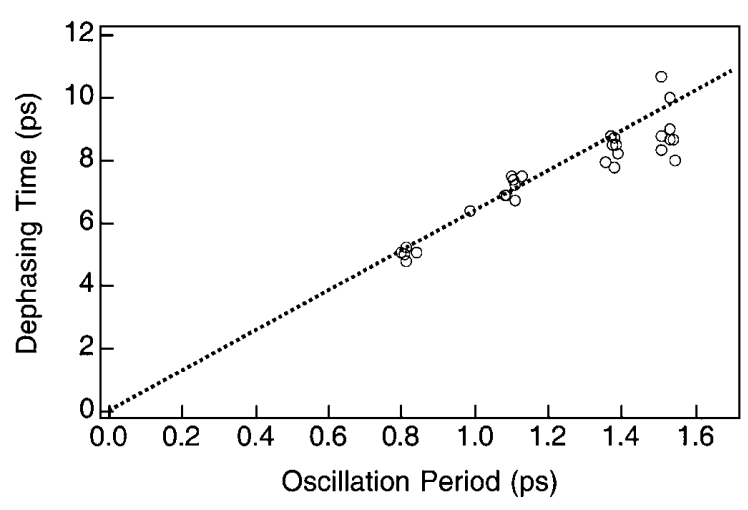

FIG. 5. Dephasing time $\tau$ vs oscillation period $T$ for different samples at different photoexcitation energies. The dotted line corresponds to a simple relation of $\tau=6.38 T$ governed by the finite sample width of 14 QWs.

We have also plotted the simple relation of $\tau=6.38 T$ as a dashed line for comparison. Excellent agreements can be found except for the thick quantum well samples with long oscillation periods. For the thick well width samples probed with higher photon energies, the absorption was so strong that the photocarriers were unevenly distributed with much higher carrier densities in the front wells facing the excitation beams. Carriers were thus distributed within an even narrower region than the sample width, creating even larger $\Delta q$ and thus shorter dephasing time for the high excitation-photon-energy data.

In summary, we have studied the coherent longitudinalacoustic phonon oscillation in $\mathrm{InGaN} / \mathrm{GaN}$ multiple quantum wells. This oscillation was initiated by photocarrier screening of the piezoelectric field via the coupling between periodic carrier population and the corresponding acoustic phonon mode. The resulted modulation of strain and piezoelectric field would then result in large optical property oscillation through quantum-confined Franz-Keldysh effect. Large strain variation and optical transmission modulation both on the order of $1 \%$ or a fraction of $1 \%$ can be easily achieved. However, because of the limited sample thickness, the magnitude uncertainty in the corresponding wave vector caused fast dephasing of the observed coherent oscillation. This easily accessible oscillation opens the way to the studies of the properties of acoustic phonons and their interactions with surrounding carriers in semiconductors. With such a large transmission modulation due to coherent acoustic phonon oscillation, this interesting phenomena not only cannot be neglected, but can be applied to practical high-speed optoelectronic applications with a tunable oscillation frequency up to the $\mathrm{THz}$ range within one material system.
The authors acknowledge the stimulating scientific discussions with Professor E.P. Ippen, Professor C. J. Stanton, Professor D.H. Reitze, and Professor Y.-M. Chang. The InGaN MQW samples were kindly provided by A. Abare and Professor S.P. DenBaars. This work is sponsored by National Science Council of Taiwan, R.O.C., under Grant No. NSC 88-2112-M-002-003. X. Y. Y. acknowledges support from National Science Council through NSC-88-2816-M-002-0002-6.

*Electronic address: sun@cc.ee.ntu.edu.tw

[1] G. C. Cho, W. Kutt, and H. Kurz, Phys. Rev. Lett. 65, 764 (1990).

[2] W. A. Kutt, W. Albrecht, and H. Kurz, IEEE J. Quantum Electron. 28, 2434 (1992).

[3] T. Pfeifer, W. Kutt, H. Kurz, and H. Scholz, Phys. Rev. Lett. 69, 3248 (1992).

[4] T. K. Cheng, J. Vidal, H. J. Zeiger, G. Dresselhaus, M. S. Dresselhaus, and E. P. Ippen, Appl. Phys. Lett. 59, 1923 (1991).

[5] W. Albrecht, T. Kruse, and H. Kurz, Phys. Rev. Lett. 69, 1451 (1992).

[6] A. V. Kuznetsov and C. J. Stanton, Phys. Rev. B 51, 7555 (1995).

[7] E. R. Thoen, G. Steinmeyer, P. Langlois, E. P. Ippen, G. E. Tudury, C. H. Brito Cruz, L. C. Barbosa, and C. L. Sedar, Appl. Phys. Lett. 73, 2149 (1998).

[8] A. Yamamoto, T. Mishina, and Y. Masumoto, Phys. Rev. Lett. 73, 740 (1994).

[9] A. Bartels, T. Dekorsy, H. Kurz, and K. Köhler, Phys. Rev. Lett. 82, 1044 (1999).

[10] S. F. Chichibu, A. C. Abare, M. S. Minsky, S. Keller, S. B. Fleischer, J. E. Bowers, E. Hu, U. K. Mishra, L. A. Coldren, S. P. DenBaars, and T. Sota, Appl. Phys. Lett. 73, 2006 (1998).

[11] S. Nakamura and G. Fasol, The Blue Laser Diode (Springer-Verlag, Berlin, Heidelberg, 1997).

[12] T. Takeuchi, S. Sota, M. Katsuragawa, M. Komori, H. Takeuchi, H. Amano, and I. Akasaki, Jpn. J. Appl. Phys., Part 2 36, L382 (1997).

[13] C.-K. Sun, J.-C. Liang, J.-C. Wang, F.-J. Kao, S. Keller, M.P. Mack, U. Mishra, and S.P. DenBaars (to be published).

[14] M. D. Fayer, IEEE J. Quantum Electron. 22, 1437 (1986).

[15] P. Basséras, S. M. Gracewski, G. W. Wicks, and R. J. D. Miller, J. Appl. Phys. 75, 2761 (1994).

[16] C.-K. Sun, F. Vallée, S. Keller, J.E. Bowers, and S.P. DenBaars, Appl. Phys. Lett. 70, 2004 (1997).

[17] V. A. Savastenko and A. U. Sheleg, Phys. Status Solidi A 48, K135 (1978).

[18] D. A. B. Miller, D. S. Chemla, and S. Schnitt-Rink, Phys. Rev. B 33, 6976 (1986). 